\title{
The Fermi Bubbles from Stochastic Acceleration by Turbulence in a Galactic Outflow
}

\section{Philipp Mertsch*}

Institute for Theoretical Physics and Cosmology (TTK), RWTH Aachen University, Germany; Niels Bohr International Academy, Niels Bohr Institute, Blegdamsvej 17, 2100 Copenhagen, Denmark;

Kavli Institute for Particle Astrophysics \& Cosmology, 2575 Sand Hill Road, M/S 29, Menlo Park, CA 94025, USA

E-mail: pmertsch@physik.rwth-aachen.de

\section{Vahé Petrosian}

Kavli Institute for Particle Astrophysics \& Cosmology, 2575 Sand Hill Road, M/S 29, Menlo Park, CA 94025, USA;

Department of Physics, Stanford University, Stanford, CA 94305, USA

E-mail: vahepestanford.edu

\begin{abstract}
The discovery of the Fermi bubbles - a huge bilobular structure seen in GeV gamma-rays above and below the Galactic center - implies the presence of a large reservoir of high energy particles at $\sim 10 \mathrm{kpc}$ from the disk. The absence of evidence for a strong shock coinciding with the edge of the bubbles, and constraints from multi-wavelength observations point towards stochastic acceleration by turbulence as a likely model of acceleration. We have investigated the time-dependent acceleration of electrons and protons in a large-scale outflow from the Galactic centre. For the first time, we present a detailed numerical solution of the transport equation that includes the relevant transport and energy loss processes. Fitting to the observed spectrum and morphology of the bubbles allows determining the parameters of the outflow, thereby shedding light on the origin of the Fermi bubbles.
\end{abstract}

35th International Cosmic Ray Conference

10-20 July, 2017

Bexco, Busan, Korea

\footnotetext{
*Speaker.
} 


\section{Introduction}

The detection of the Fermi bubbles-a huge bi-lobular structure seen in GeV gamma-rays-is certainly one of the great discoveries made with the Fermi-LAT instrument. Due to their position on the sky (see Fig. 1 and Sec. 2.1 below), they are likely emanating from the Galactic centre and the most speculated about sources are the supermassive black hole at the Galactic centre and star formation/star burst in the Galactic centre region. These processes shape Galactic structure on the largest scales and as such the Fermi bubbles allow us to study Galactic feedback in our own backyard. Furthermore, given their prominence in gamma-rays, they are an important background for all kinds of diffuse studies at $\mathrm{GeV}$ energies, like searches for signals from self-annihilation or decay of dark matter. Finally, the formation of the gamma-rays and the acceleration of the underlying cosmic rays are of astrophysical interest in itself.

\section{Observations}

\subsection{Properties in gamma-rays}

Originally the Fermi bubbles were observed in a search [1] for the gamma-ray counterpart of a microwave excess seen from the inner Galaxy [2, 3, 4]. A more detailed analysis [5] unveiled some surprising properties that were later largely confirmed [6] by the Fermi collaboration:

Geometry: The Fermi bubbles are approximately centered at zero Galactic longitude, symmetric about the Galactic plane, $50^{\circ}$ wide in longitude and each bubble extends up to $50^{\circ}$ in latitude, see Fig. 1. On these scales, they constitute the first evidence for an outflow from the Milky Way. (On smaller scales, there had previously been evidence in X-rays, in particular an X-shaped feature around the Galactic centre.) The bubbles' symmetry about the Galactic plane and their being

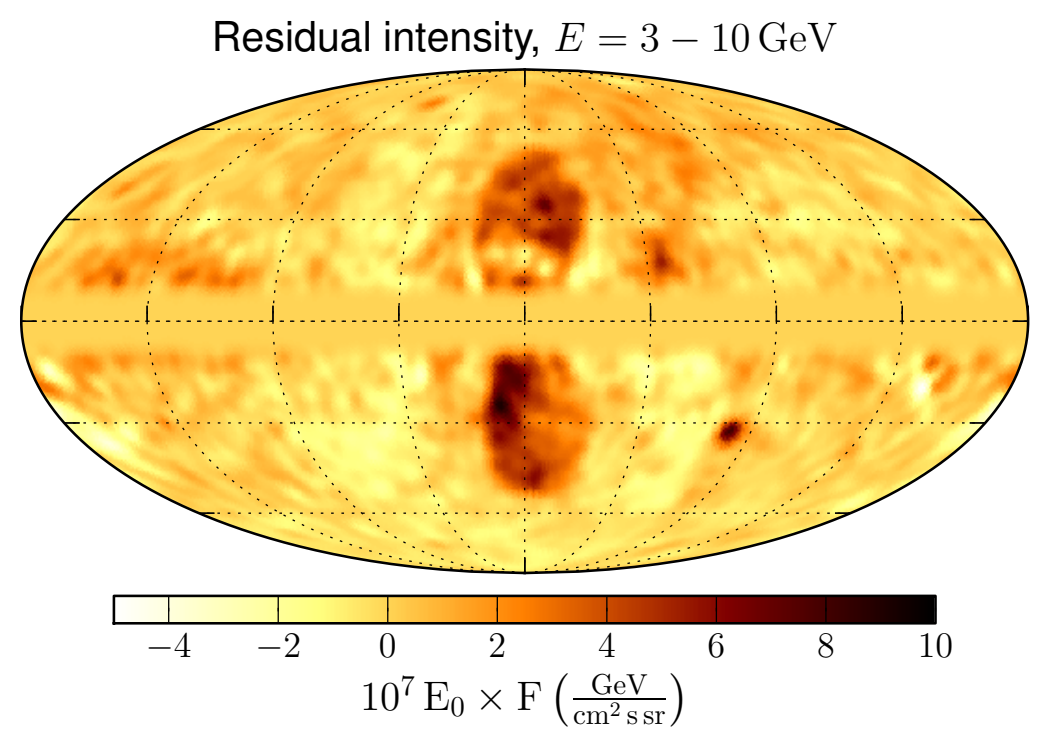

Figure 1: Sky map of the Fermi bubbles. Shown is the residual in $3-10 \mathrm{GeV}$ gamma-rays after template subtraction. See Ref. [6] for details of the analysis. 
centred around zero longitude imply a Galactic centre origin. A wind with a constant speed of $1000 \mathrm{~km} \mathrm{~s}^{-1}$ would need about $9.9 \mathrm{Myr}$ to expand the bubbles up to $d_{G C} \tan 50^{\circ} \simeq 10.1 \mathrm{kpc}$ above the Galactic centre which is at a distance $d_{G C} \simeq 8.5 \mathrm{kpc}$. Note however projection effects: At a latitude of $50^{\circ}$, we might be seeing the limb-brightened edge of a bubble of radius $d_{G C} \sin 50^{\circ} \simeq 6.5 \mathrm{kpc}$, thus reducing the time-scale to $6.4 \mathrm{Myr}$. Note that the eastern edge of the northern bubble is very close to the position of the North-polar spur, which is part of the the radio Loop I. Initially, this led to claims of the bubbles being associated with the Loop I structure [7].

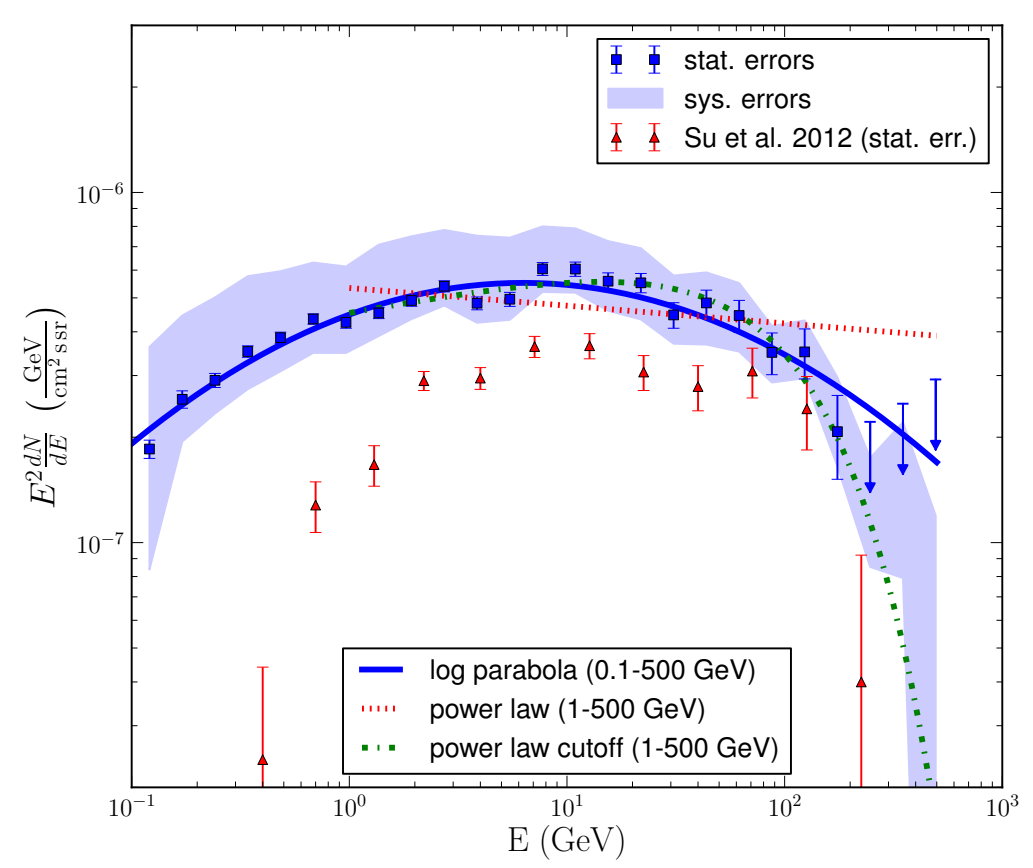

Figure 2: Overall spectrum of the Fermi bubbles, that is the average diffuse flux, multiplied with photon energy squared $E^{2}$. Statistical errors are shown by the error bars, systematic uncertainties, due to the uncertainty in the Galactic diffuse modelling are indicated by the shaded band. Instead of specific hadronic or leptonic models, three parametric fits to the spectrum are shown: a log-parabola, a simple power law and a power law with an exponential cut-off. See Ref. [6] for the inferred parameters.

Spectrum: The gamma-ray flux shows a hard spectrum, mostly $\propto E^{-2}$ and extending from a few hundred $\mathrm{MeV}$ up to a few hundred $\mathrm{GeV}$, see Fig. 2. At lower energies, the spectrum is significantly harder and above, there is evidence for a spectral softening or an exponential cut-off. This spectral shape immediately invites speculation about its physical origin, i.e. relating to the question whether the gamma-rays are of leptonic, inverse-Compton scattering or hadronic, $\pi^{0}$ emission origin. (Given the estimates of the gas density inside the bubbles, see below, bremsstrahlung is likely negligible.)

While the spectral shoulder around a few hundred $\mathrm{MeV}$ determined in the earlier analysis [5] seemed to be well fit by the kinematic feature from $\pi^{0}$ emission, the new best-fit spectrum appears to be extending to lower energies. Likely, a hadronic model needs to have a spectral break at low 
energies in the underlying proton spectrum. In addition the spectral break or cut-off at high energies requires an additional break. The physical origin of the breaks is a priori unclear.

In leptonic models these breaks are easily explained. The inverse-Compton spectrum is naturally rather hard: In the Compton regime, an electron spectrum $\propto E^{-\Gamma}$ produces a gamma-ray spectrum $\propto E^{-s}$ with $s=(\Gamma+1) / 2$. For a hard electron spectrum with a cut-off at an energy $E_{\text {cut }}$, the gamma-ray spectrum from scattering of a radiation field with characteristic energy $\varepsilon$ results in a peak around $\left(E_{\text {cut }} / m_{\mathrm{e}} c^{2}\right)^{2} \varepsilon$ in gamma-ray energy. The apparent $E^{-2}$ spectrum could then be explained as the sum of the Inverse-Compton scattering on CMB, IR and optical/UV backgrounds. An $E_{\text {cut }} \sim 500 \mathrm{GeV}$ could explain the spectral breaks at a few hundred $\mathrm{MeV}$ and a few hundred $\mathrm{GeV}$ and would thus naturally explain the range of the spectrum in energy.
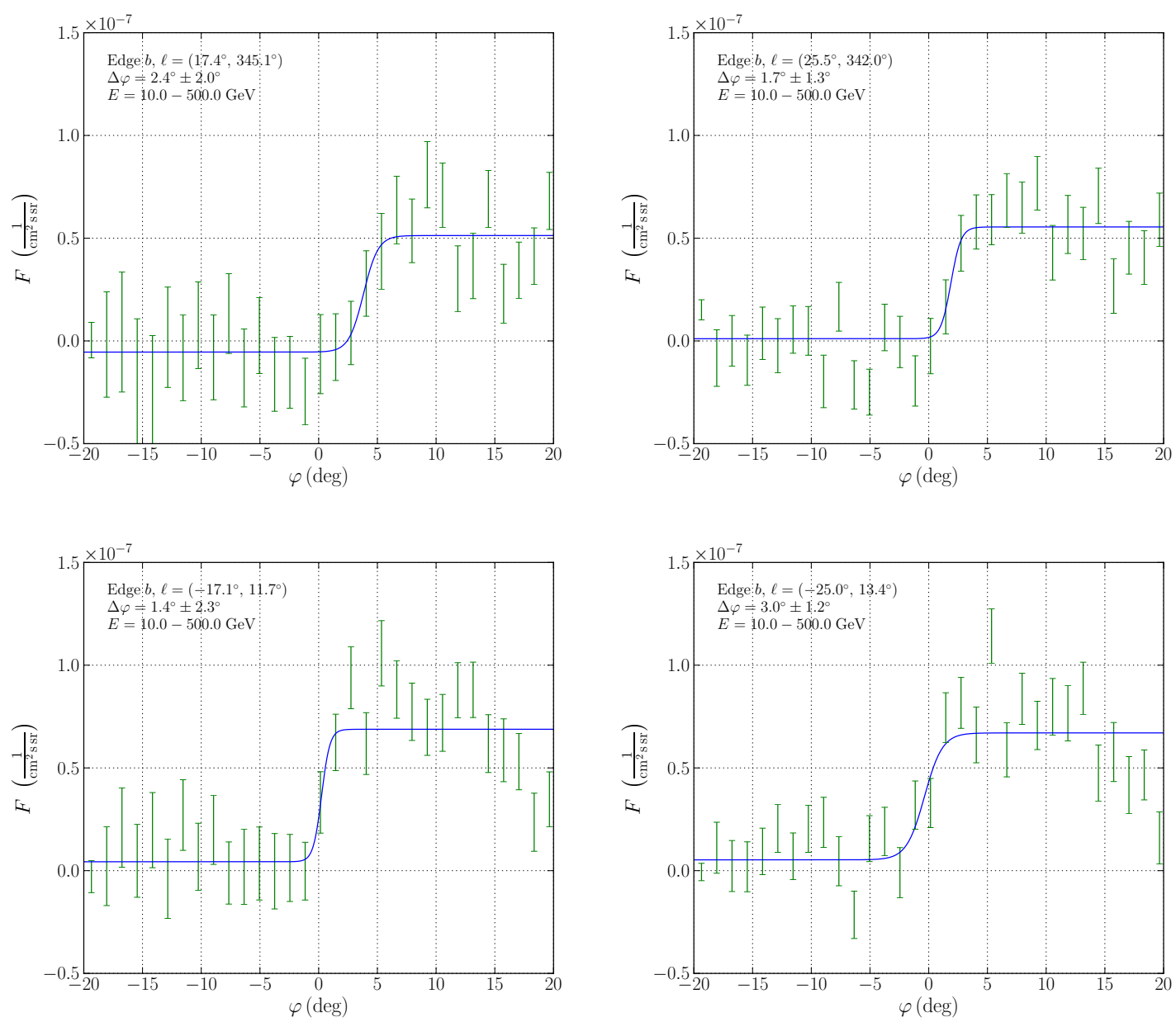

Figure 3: Flux profiles along the gradient direction across the bubble edge at four different position. To guide the eye, a hyperbolic tangent function is fitted to the data, $\propto \tanh \left(\left(\phi-\phi_{0}\right) / \Delta \phi\right)$, with the characteristic width $\Delta \phi$ indicated in the legends. From Ref. [6]

Surface brightness. The surface brightness shows little variation over the bubbles, but has sharp edges as can already be seen in the residual map, cf. Fig. 1. More quantitatively, this is evidenced by profiles of the gamma-ray flux across the bubble edge, shown in Fig. 3. There is clearly a jump in 
intensity from a value close to zero (after template subtraction) outside to a relatively constant value inside the bubbles. In fact, the only substructure seen is a rather large enhancement of emissivity in the east of the southern bubble, see Fig. 1, called the "cocoon", the origin of which is of yet unknown. There have also been claims of evidence for a narrow and extendend, jet-like feature [8], however, the analysis by the Fermi collaboration [6] has found this feature not to be significant.

The flat surface brightness and sharp edges are one of the most puzzling features of the bubbles. Not only do the sharp edges imply an efficient confinement of the gamma-ray producing cosmic rays. In addition, the flat surface brightness requires a peculiar distribution of emissivity: Idealising each bubble as a spherically symmetric volume with of outer radius $R$, only an emissivity that varies with radius $r$ as $1 / \sqrt{1-(r / R)^{2}}$ will give a flat surface brightness and sharp edges.

Spectral uniformity. The bubbles show similar morphologies in different energy bins ranging from 1 to $500 \mathrm{GeV}$ (see Fig. 4) and equivalently the spectrum is uniform in different parts of the bubbles. Specifically, the gamma-ray spectrum has been analysed in different latitude bands and the spectrum in the bubble edge region and the interior have been compared: For the latitude bands, no variation has been found above and below $\pm 10^{\circ}$. Between $-10^{\circ}$ and $+10^{\circ}$ there is an excess at the Galactic Centre [9], likely with a spherical symmetry, and its connection to the Fermi bubbles is unclear at this point [10]. Furthermore, no variation between the edge region and the interior was found [5] (but see Ref. [11]).

The spectral uniformity is also very surprising for such an extended structure. Leptonic models in particular would be expected to lead to some variation, depending on the region of energisation of the high-energy electrons. This is due to cooling losses by synchrotron radiation and inverse-Compton emission. A conservative estimate of the cooling time is $\tau_{\text {cool }}=6.74 \times$ $10^{8} \mathrm{yr}(E / \mathrm{GeV})^{-1}\left(\left(u_{B}+u_{\mathrm{CMB}}\right) /\left(0.486 \mathrm{eVcm}^{-3}\right)\right)^{-1}$, for magnetic fields and radiation fields of energy densities $u_{B}=0.224 \mathrm{eV} \mathrm{cm}^{-3}(B / 3 \mu \mathrm{G})^{2}$ and $u_{\mathrm{CMB}}=0.262 \mathrm{eV} \mathrm{cm}^{-3}$, respectively, i.e. of the same order as the bubble age for $100 \mathrm{GeV}$ electrons. Therefore, electrons energised in the Galactic plane will be subject to considerable cooling while travelling out into the bubble volume. This results in softer spectra at larger distances from the Galactic centre and thus a softer gamma-ray spectrum at higher latitudes. In addition, the energy densities in the radiation backgrounds that the electron inverse-Compton scatter on should be varying with distance from the disk: While the CMB is of course spatially uniform, the energy densities in both the optical/UV and the infrared backgrounds should become smaller further away from the disk. The fact that this is not observed imply that the variation in the radiation backgrounds must be counter-balanced by a variation in the electron spectrum to some degree.

\subsection{Hints}

While the discovery of the Fermi bubbles was certainly a surprise, it was not the first hint at the presence of Galaxy-scale outflows. Kiloparsec-scale outflows have been observed for starburst galaxies, e.g. in ionised gas. In Fig. 5, we show NGC3079 in $\mathrm{H} \alpha$. Multiple shells emanating from the centre of NGC3079 are visible.

Even in our own Milky Way, there had been hints at the presence of a Galaxy-scale outflow, possibly connected with high-energy cosmic rays: Observations in soft X-rays, most notably from 

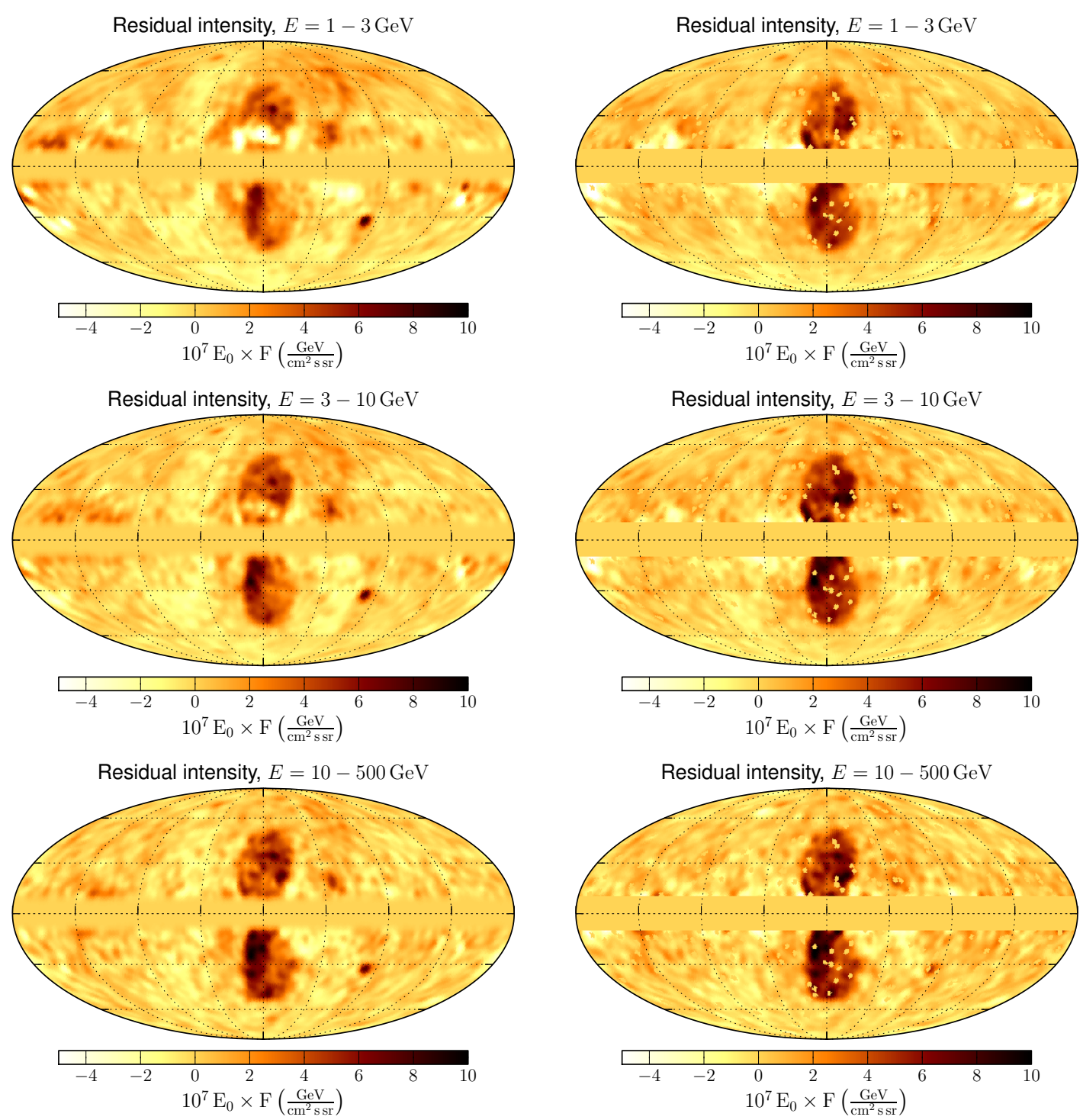

Figure 4: Residual intensities for different energies and two different methods of foreground cleaning. Left column: Using gas maps and a GALPROP model for the standard Galactic inverse-Compton contribution. Energy bins of $1-3 \mathrm{GeV}$ (top), $3-10 \mathrm{GeV}$ (middle) and $10-500 \mathrm{GeV}$ (bottom panel). Right column: Using gas maps and locally Gaussian templates for the standard Galactic inverse-Compton contribution. Energy bins of $1-3 \mathrm{GeV}$ (top), $3-10 \mathrm{GeV}$ (middle) and $10-500 \mathrm{GeV}$ (bottom panel). From Ref. [6]

ROSAT, showed signs of an $\mathrm{x}$-shaped feature, interpreted as evidence of a biconical outflow in analogy with structures seen in other galaxies.

The presence of a population of high-energy cosmic ray electrons was already hinted at by the microwave haze, an excess of microwaves from the Galactic centre, pointing at a similarly hard electron spectrum [2, 3, 4]. (Note the possible influence of systematic effects due to template subtraction [13], however.) The search for a counterpart of the microwave haze in gamma-rays was in fact what motivated the first study that showed evidence for an extended gamma-ray emission [1]. 


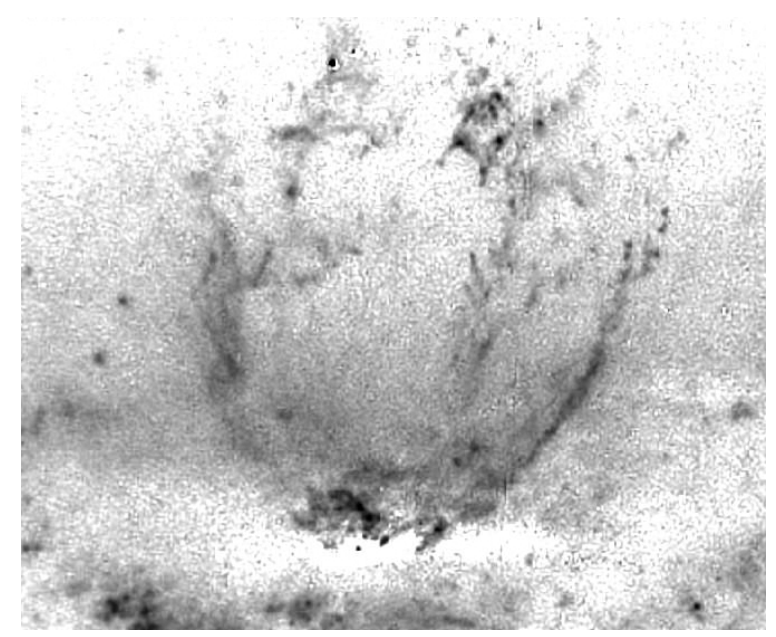

Figure 5: Superbubble(s) in NGC 3076, seen in $\mathrm{H} \alpha$. The (outer) bubble is $1 \times 1.2 \mathrm{kpc}$ in size. From Ref. [12].

\subsection{Other constraints}

X-rays. A number of studies have investigated the properties of the thermal gas in the Fermi bubbles and in the Galactic halo from X-ray observations. The parameters can be either inferred from the thermal, soft X-ray spectrum $[14,15]$ or from individual Oxygen lines [16]. The gas densities inferred are of the order $n_{\text {gas }} \sim 10^{-3} \mathrm{~cm}^{-3}$ and the temperatures of the gas just outside the bubbles vary between $k T \simeq 0.3 \mathrm{keV}$ and $0.5 \mathrm{keV}$. This is higher than the canonical temperature of the Galactic halo of $k T \simeq 0.2 \mathrm{keV}$ and this is interpreted as shock heating. This implies Mach numbers of the shock of $\mathscr{M} \simeq 1.5 \ldots 2.3$, that is rather weak shocks. Finally, with the typical sound speed in the Galactic halo of $c_{s} \simeq 200 \mathrm{~km} \mathrm{~s}^{-1}$, one infers shock speeds of $v \simeq 300 \ldots 500 \mathrm{~km} \mathrm{~s}^{-1}$.

The absence of evidence for a strong shock coinciding with the bubble edge is somewhat puzzling and probably implies that diffusive shock acceleration at the bubble edge cannot be efficient. If electrons get accelerated in the Galactic plane or even in a hypothetical large-scale jet along the Galactic minor axis, they need to travel over kiloparsec distances to fill the bubble volume and thus suffer from sever cooling losses. These would significantly soften the spectrum, if not quench the electron density completely. Note further that the low shock speeds found by the X-ray modelling lead to even larger dynamical times than with the $1000 \mathrm{~km} \mathrm{~s}^{-1}$ assumed above, making the energy losses even more severe.

Quasar absorption. The observation of absorption by the gas associated with the bubbles from a background quasar can also be used to set bounds on the outflow speed. In the UV absorption lines from PDS 456 two (asymmetric) components with velocities of $v \simeq-235$ and $+250 \mathrm{~km} \mathrm{~s}^{-1}$ with respect to the local standard of rest could be identified [16]. For the conical outflow assumed in that study, this implies an upper limit on the outflow speed of $\gtrsim 900 \mathrm{~km} \mathrm{~s}^{-1}$. This seems to be in conflict with the shock speed inferred from the X-ray modelling (see above). Note, however, that the outflow speed inferred from the absorption lines of one quasar is very dependent on the assumed geometry of the flow. Future observations of additional sight lines towards other quasars can help mapping out the flow structure, thus possibly also constraining it geometry, and might 
bring the results into agreement with the values inferred from X-rays.

\section{Models}

The Fermi bubbles have also generated a great deal of interest on the modelling side. The variety of models is most conveniently classified by:

- the source of energy: super massive black hole or stellar winds/supernovae;

- the acceleration region: jet or sources in the disk or in situ (by shocks or turbulence);

- the nature of the high-energy particles: hadrons or leptons.

(Of course, the individual options are not mutually exclusive. For instance, in hadronic models, the bulk of the high-energy gamma-rays comes from decay of neutral pions. Charged pions, however, get produced at similar rates and can, given the radiation fields, inverse-Compton scatter into gamma-rays, mostly contributing at lower energies.) As a full discussion of all proposed models is beyond the scope of this highlight presentation, only two particular classes of models will be presented, and a few concrete examples will be shown.

Jet models. Astrophysical jets are thought to be powered by accretion onto a spinning, compact object, like neutron stars or black holes. Given the position and symmetry of the Fermi bubbles, the supermassive black hole at the Galactic centre is a prime candidate. Although conspicuously quiet (its X-ray luminosity is currently more than 11 orders below the Eddington luminosity), there is indirect evidence for earlier epochs of active accretion, e.g. from X-ray reflections.

Jets are usually associated with high speeds $\gtrsim 1000 \mathrm{~km} \mathrm{~s}^{-1}$. This allows for the electrons to be less impacted by energy losses than in starburst/star formation models and therefore the source of energisation of the high-energy electrons can be in the Galactic disk or inside the jet. (Note, however, that the jet speed is not necessarily directly implying the dynamical age as the bubbles can be formed by a fountain-like back flow due to the termination of the jet by the ram pressure of the Galactic halo.)

One of the earliest studies of a leptonic jet model employing a hydrodynamical simulation [17] found that the lateral extent of the Fermi bubbles could be explained if the jet was underdense but slightly overpressured. If active at $10 \%$ of the Eddington luminosity for $1-2 \mathrm{Myr}$ until about a Myr ago, the morphology would match the observations. A subseqent MHD simulation of the Fermi bubbles blown up by a jet [18] showed further that the shock compression at the bubble edges would compress the magnetic field such that it gets aligned with the bubble edge. We will return to this point in Sec. 4.

Star formation/star burst models. The Galactic winds that get collectively powered by an ensemble of stellar winds or supernova activity, are usually operating at smaller speeds, $\lesssim 500 \mathrm{~km} \mathrm{~s}^{-1}$. This implies larger time-scales than for the jet models leading to a preference in the literature for hadronic models, as leptons would cool too much. In hadronic models, on the other hand, cosmic rays need to be accumulated over much longer time scales, given the low gas densities of the order of $10^{-3} \mathrm{~cm}^{-3}$ (see Sec. 2.3), to produce the same gamma-ray fluxes. In turn, this and the observed 
hard $E^{-2}$ spectrum require an effective confinement of the high-energy cosmic rays to the bubbles and a suppression of (energy-dependent) escape. (See Ref. [11], however.) The sources are nevertheless oftentimes assumed to be in the Galactic disk.

The most detailed numerical star formation/star burst model for the Fermi bubbles as of yet [19] employs a hydrodynamical code to investigate the interaction of a Galactic wind with the circumgalactic medium. It is found that a luminosity of $5 \times 10^{40} \mathrm{erg} \mathrm{s}^{-1}$ and a density in the halo of $10^{-3} \mathrm{~cm}^{-3}$ can reproduce the morphology observed in gamma-rays and is also in agreement with X-ray observations. Interestingly, this luminosity is close to the one inferred from the current star formation rate, $\mathrm{SFR} \simeq 0.007 \ldots 0.1 M_{\odot} \mathrm{yr}^{-1}$, when assuming an efficiency of $30 \%$ for conversion into mechanical power, $\mathscr{L} \simeq 10^{40} \mathrm{erg} \mathrm{s}^{-1} \varepsilon_{0.3}\left(\mathrm{SFR} /\left(0.1 M_{\odot} \mathrm{yr}^{-1}\right)\right)$.

The outflow from the inner Galaxy leads to a shock structure known from astrospheres or supernova remnants, with a radial forward shock at $\sim 11 \mathrm{kpc}$, a more tangled contact discontinuity extending to $\sim 8 \mathrm{kpc}$ above the Galactic centre and a very much tangled reverse shock a few kiloparsecs inside of the contact discontinuity. Thus in this model the edge of the gamma-ray bubbles does not coincide with the projection of the forward shock, but rather the contact discontinuity. Whether this is due to the diffusion prescription of Ref. [19] changing across the contact discontinuity would need to be explored though.

\section{Stochastic Acceleration by Turbulence in a Galactic Outflow}

Most of the models presented today, including those discussed in Sec. 3, do not model the spectrum kinetically. Instead the spectrum is assumed fixed by the acceleration process, oftentimes just set to an $E^{-2}$ spectrum, and then further assumed not to change during transport. Yet, there is a variety of process that can change the spectral shape: rigidity-dependent diffusion, acceleration at shocks, stochastic acceleration and, most importantly, energy losses. Through energy-dependent transport parameters, the spectral modelling also affects the morphology. Therefore, kinetic modelling is crucial for a reliable leptonic model. In addition, many of the models above have ignored the peculiar morphological properties of the bubbles and the undelying non-trivial distribution of morphology required to produce this. Both these shortcomings are addressed in our model.

The absence of evidence for a strong shock has motivated us to revisit the 2nd-order Fermi acceleration model of the Fermi bubbles. In the pioneering work of Ref. [20], the electron spectrum was computed analytically by using a hierarchy of times-scales: For the parameters adopted the dynamical time was found to be always larger than the acceleration and energy-loss times, such that the electron spectrum would be in a quasi steady-state at fixed distances from the (expanding) shock. This required ignoring diffusion as a spatial transport process. In addition, the adiabatic term in the transport equation was absent (implying also the neglect of shock acceleration). Finally, the radiation background for energy losses and production of gamma-rays by inverse-Compton scattering was fixed to a benchmark value at $5 \mathrm{kpc}$ above the Galactic centre.

The model we consider now is much more general: We solve the full transport equation for the (isotropic) phase space density $f(\vec{r}, p, t)$, e.g. [21],

$$
\frac{\partial f}{\partial t}=\nabla \cdot(K \cdot \nabla f-\vec{V} f)+\frac{1}{p^{2}} \frac{\partial}{\partial p}\left(p^{2} D_{p p} \frac{\partial f}{\partial p}\right)+\frac{1}{p^{2}} \frac{\partial}{\partial p} p^{2}\left(\frac{p}{3}(\nabla \cdot \vec{V}) f\right)+q .
$$


Here, spatial transport is governed by the diffusion tensor $K$ and the advection velocity $\vec{V}$, the latter also leading to adiabatic gains/losses through the divergence term. Momentum space diffusion depends on the diffusion coefficient $D_{p p}$. This equation does not hold at the shock where the velocity field is discontinuous. Therefore, we derive a shock equation by demanding continuity of phase space density and phase space flux across the shock. The results from the MHD simulations discussed in Sec. 3 have shown the formation of a shock emanating from the Galactic centre, with the large-scale magnetic field aligned with it. We match this morphology by assuming toroidal coordinates $(u, v, \phi)$ with a pseudo-radial coordinate $u$ and a pseudo-polar coordinate $v$, and demanding the shock to be on iso contours in $u$. This has the added benefit of rendering the diffusion tensor diagonal. Furthermore, we demand azimuthal symmetry $(\partial / \partial \phi \equiv 0)$. The shock is strong only along the Galactic minor axis along which the expansion speed is set to a constant $V_{\mathrm{sh}}$. Electrons get steadily injected with a momentum $p c=10^{-2} \mathrm{GeV}$ at the Galactic centre. The ensuing equations are solved by a finite difference method and the parameter values adopted are summarised in Tbl. 1. See Fig. 6 for a schematic overview of the three models, but refer to the text below for explanations.
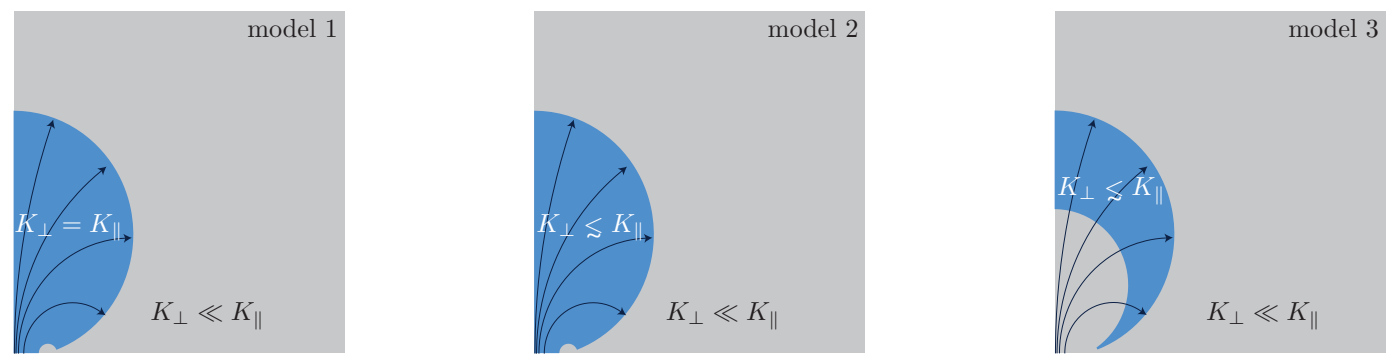

Figure 6: Schematic overview of the three models. (See text for explanations.)

\begin{tabular}{|c|c|c|c|c|c|c|c|}
\hline & \multicolumn{2}{|c|}{ model 1} & \multicolumn{2}{|c|}{ model 2} & \multicolumn{3}{|c|}{ model 3} \\
\hline & inside & halo & inside & halo & inside & shell & halo \\
\hline$K_{\text {uu }, 0}\left[10^{-7} \mathrm{kpc}^{2} \mathrm{yr}^{-1}\right]$ & 1 & 0.1 & 1 & 0.1 & 0.1 & 1 & 0.1 \\
\hline$K_{v v, 0}\left[10^{-7} \mathrm{kpc}^{2} \mathrm{yr}^{-1}\right]$ & 1 & 10 & 10 & 100 & 100 & 10 & 100 \\
\hline$D_{p p, 0}\left[10^{-7}(\mathrm{GeV} / c)^{2} \mathrm{yr}^{-1}\right]$ & 10 & 1 & 10 & 1 & 1 & 10 & 1 \\
\hline$\delta$ & \multicolumn{2}{|c|}{$1 / 3$} & \multicolumn{2}{|c|}{$1 / 3$} & \multicolumn{3}{|c|}{$1 / 3$} \\
\hline$V_{\mathrm{sh}}\left[10^{-7} \mathrm{kpc} \mathrm{yr}^{-1}\right]$ & \multicolumn{2}{|c|}{1} & \multicolumn{2}{|c|}{1} & \multicolumn{3}{|c|}{1} \\
\hline$r$ & \multicolumn{2}{|c|}{4} & \multicolumn{2}{|c|}{4} & \multicolumn{3}{|c|}{4} \\
\hline
\end{tabular}

Table 1: Summary of parameter choices in models 1, 2 and 3.

\subsection{Model 1}

We start with the simple model 1: isotropic diffusion inside the bubbles, anisotropic diffusion in the Galactic halo. In quasi-linear theory this choice also implies different values for the momentum diffusion coefficient $D_{p p}$ inside and outside the bubbles. The spectral index $\delta$ of the spatial 
diffusion coefficients is $\delta=0.5$ and we fix the shock speed to $10^{-7} \mathrm{kpc}$ yr. We refer the interested reader to Ref. [22] for details.

In the top panel of Fig. 7, we show the electron SED $p^{4} f$ at four different electron energies $p c=1,10,10^{2}$ and $10^{3} \mathrm{GeV}$ at time $t=2.4 \times 10^{7} \mathrm{yr}$. Whereas at low energies, the electrons are very much confined to the Galactic centre region at higher energies they extended up to and beyond the shock. In the lower panel of Fig. 7, we show the gamma-ray maps produced by inverseCompton scattering of the electrons. It can be seen that the bubbles are centre-brightened unlike the observed bubbles.
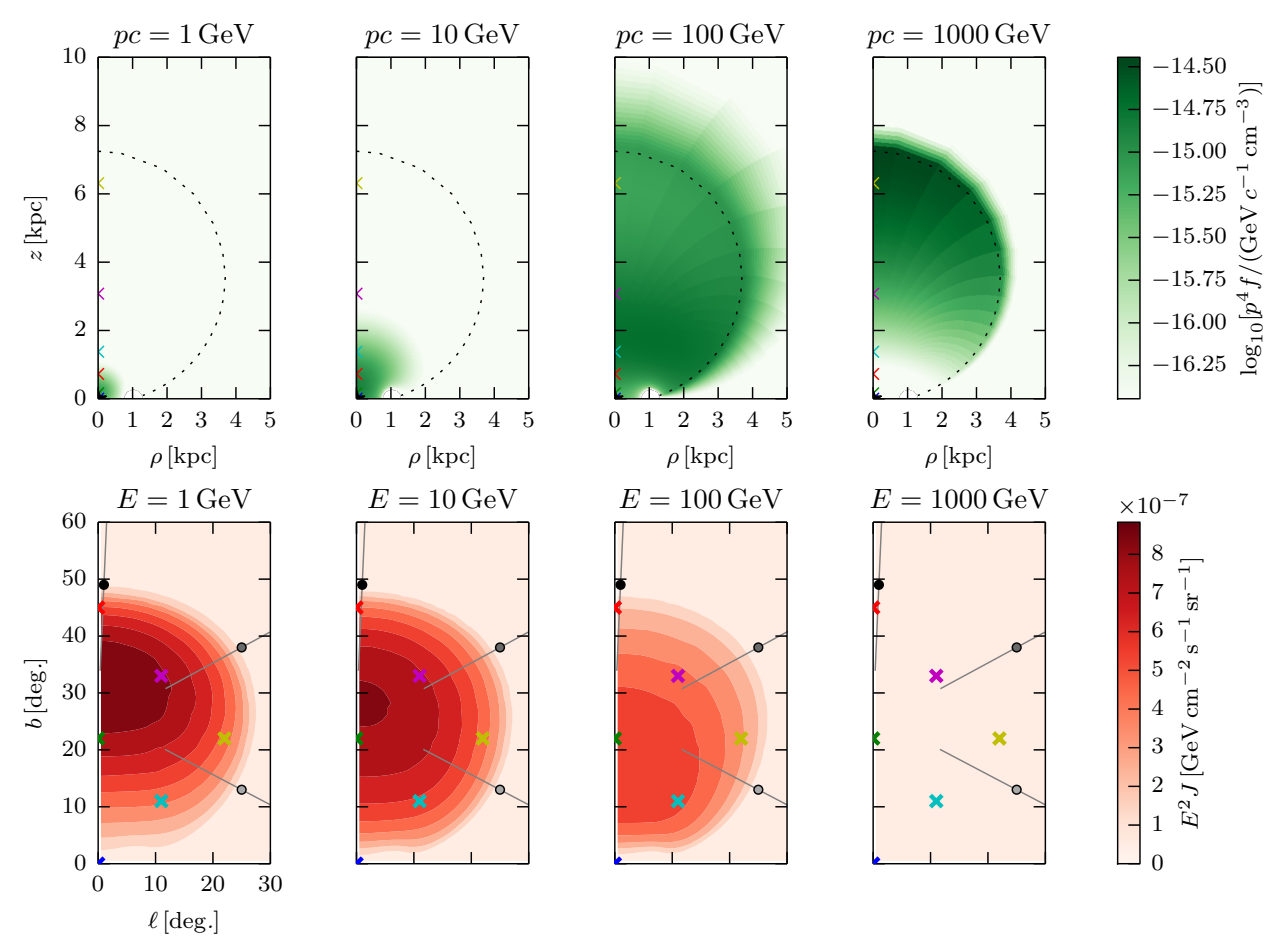

Figure 7: Top: The distribution of electron energy $p^{4} f$ at energies $p c=1,10,10^{2}$ and $10^{3} \mathrm{GeV}$ at time $t=2.4 \times 10^{7} \mathrm{yr}$. The black star marks the position of the source where electrons get steadily injected with a momentum $p c=10^{-2} \mathrm{GeV}$. Bottom: The gamma-ray maps from inverse-Compton scattering of the electrons at gamma-ray energies $E=1,10,10^{2}$ and $10^{3} \mathrm{GeV}$ at time $t=2.4 \times 10^{7} \mathrm{yr}$.

\subsection{Model 2}

In the second model, we try to modify the electron morphology in order to make the gammaray map more limb-brightened. We achieve this by making diffusion anisotropic also in the bubbles. In Fig. 8 we show the electron distribution and the gamma-ray maps for this model. In the left panel of Fig. 9, we now also show the angular profiles across the bubble edge, similarly to Fig. 3 for the observations which we also show. In the right panel of Fig. 9, we show the gamma-ray spectrum for different directions through the bubbles and compare with the data of Fig. 2. It can be seen that while our model reproduces the overall spectrum rather nicely, the angular profiles are still not sharp enough. 

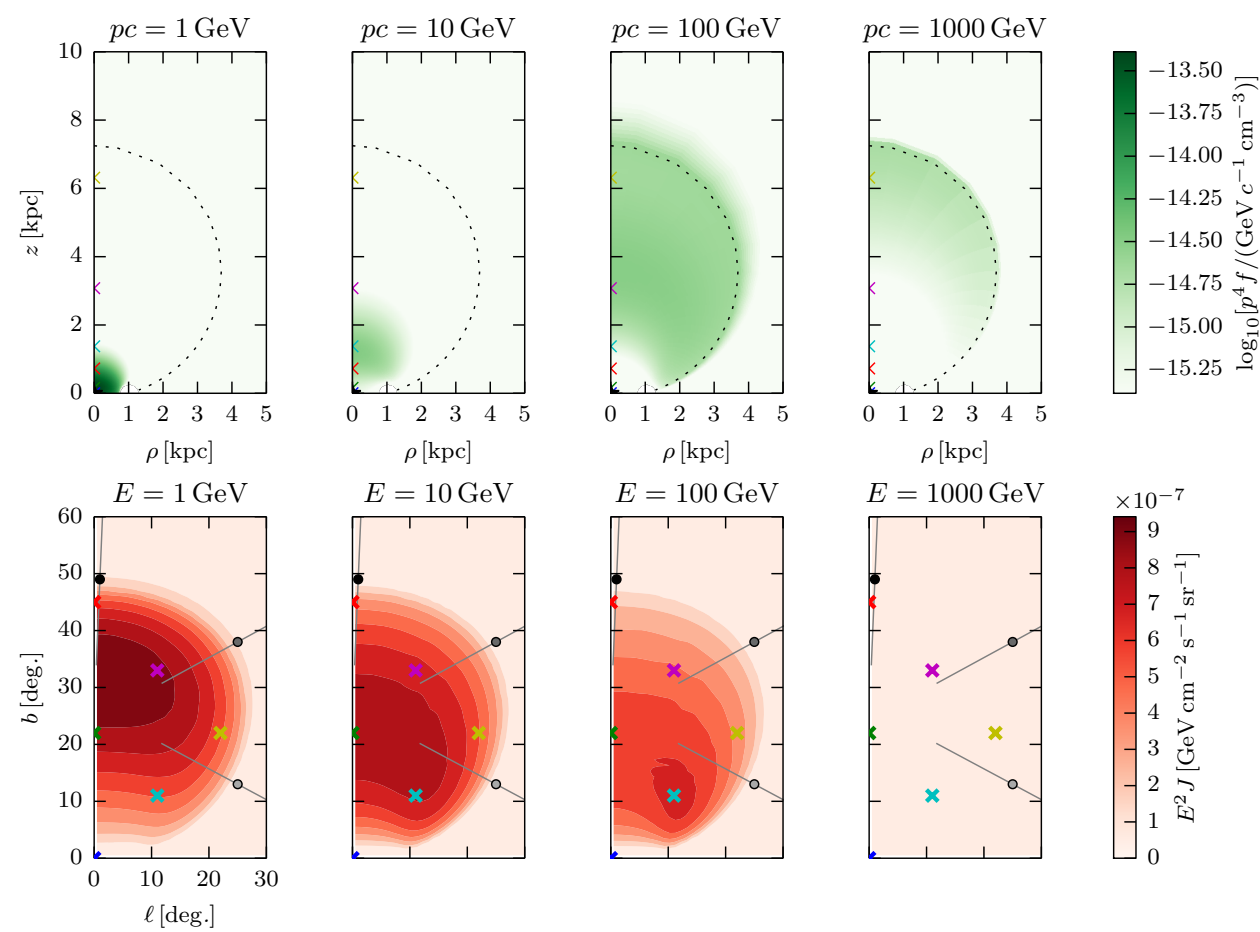

Figure 8: Same as Fig. 7, but for model 2 (homogeneous bubble, anisotropic diffusion).
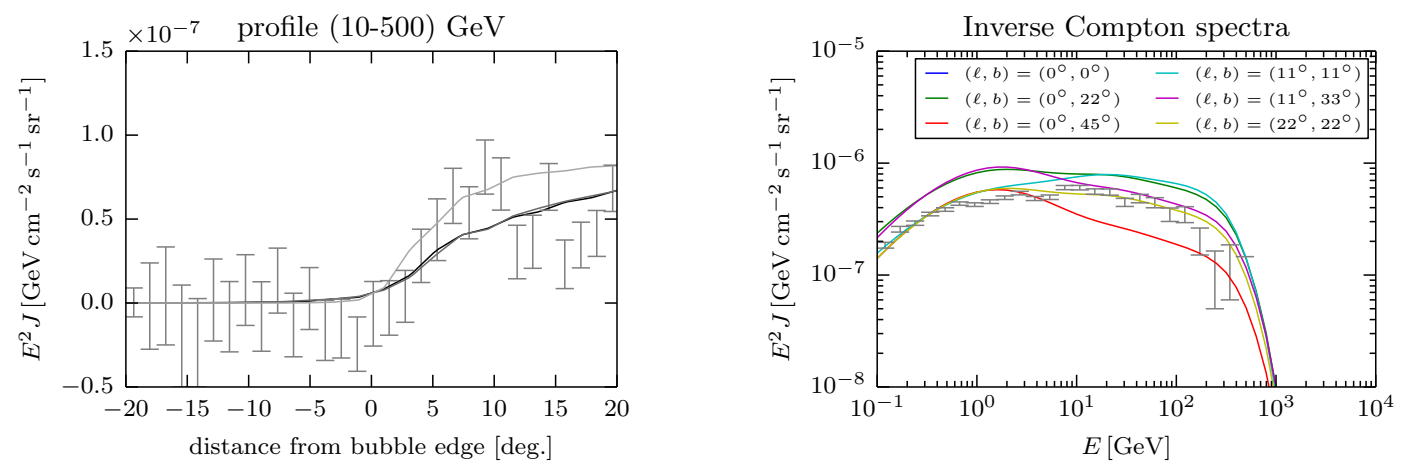

Figure 9: Left: Angular profiles across the bubble edge for gamma-rays between 10 and $500 \mathrm{GeV}$, compared with a subset of the data from Fig. 3. Right: Gamma-ray spectra for the different directions indicated in the legend and marked by the crosses in the lower panel of Fig. 8. The data are from Fig. 2.

\subsection{Model 3}

In this third model, we allow diffusion to be mildly anisotropic inside a shell around the bubble edge and strongly anisotropic both in the rest of the bubble volume and in the Galactic halo. This can be justified if turbulence was predominently generated at the shock by various instabilities and would decay as it travels into the bubble interior with the downstream bulk flow. We again refer the interested reader to Ref. [22] for details.

In Figs. 10 and 11, we show the modelled electron distribution, gamma-ray sky map, gammaray angular profiles and gamma-ray spectra in a similar way as before. It can bee seen that the 
energy distribution $p^{4} f$ of electrons peaks at high energies and close to the shock. This produces a gamma-ray sky map that is not centre-brightened any more. The predicted angular profile across the bubble edge nicely reproduces the observations and the overall spectrum is also in agreement with the data, with spectral variation between different directions through the bubbles rather small.
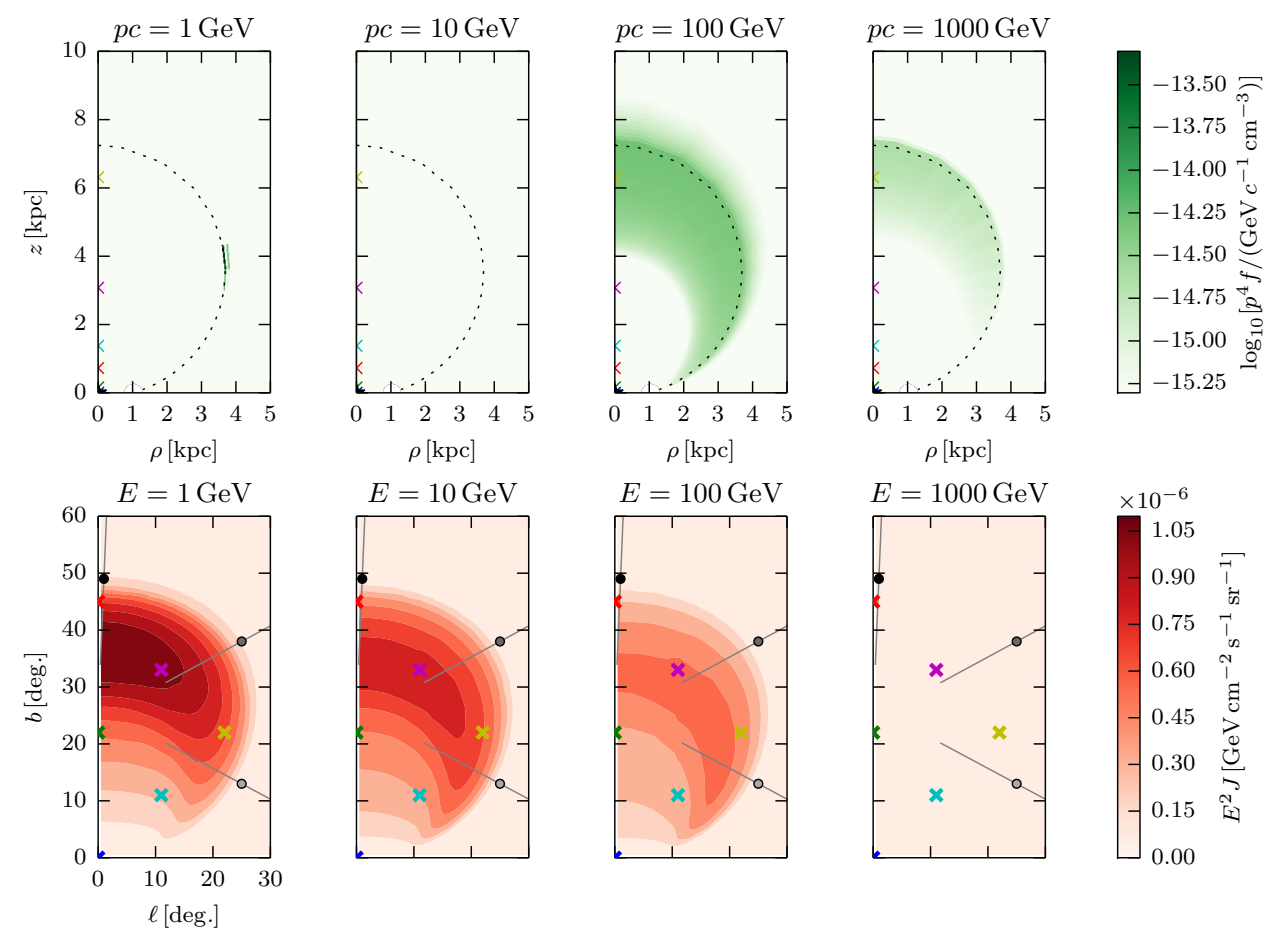

Figure 10: Same as Fig. 7, but for model 3 (bubble with shell, anisotropic diffusion).
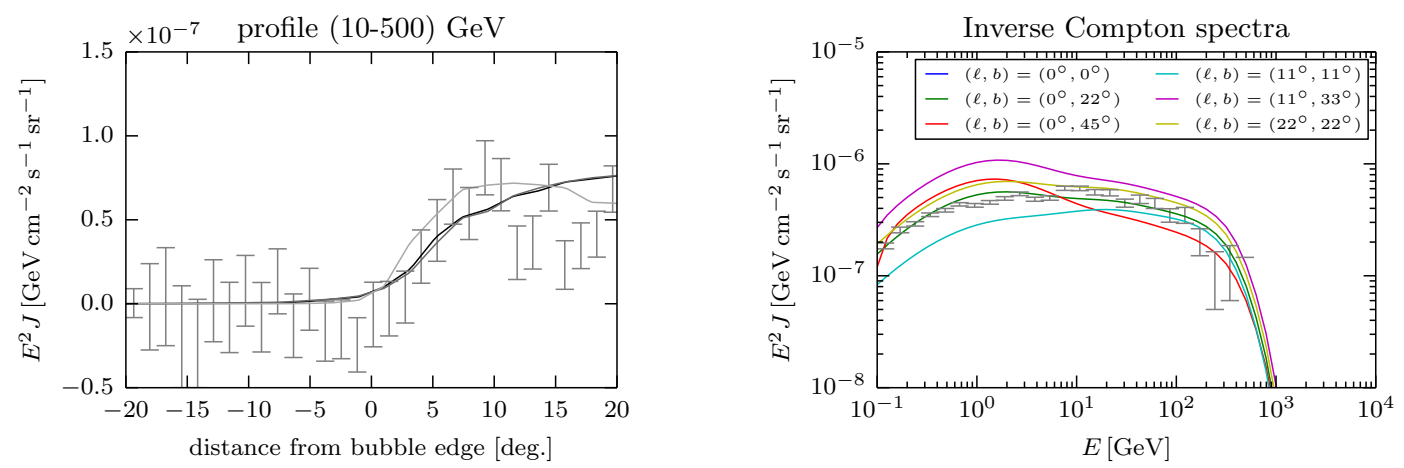

Figure 11: Same as Fig. 9, but for model 3 (bubble with shell, anisotropic diffusion).

In the upper panels of Fig. 12, we show the synchrotron intensity maps produced under the assumption of a simple exponential model for the Galactic magnetic field (RMS) value: $B_{r m s}=$ $B_{0} \exp \left[-r / r_{0}-z / z_{0}\right]$ with $r_{0}=5 \mathrm{kpc}$ and $z_{0}=2 \mathrm{kpc}$. We note that the morphology is markedly different from the gamma-ray morphology but largely in agreement with the observed microwave excess [4]. In the lower panel, we also show the radio/microwave spectra of the bubbles and compare to data from Refs. [3] and [4]. 

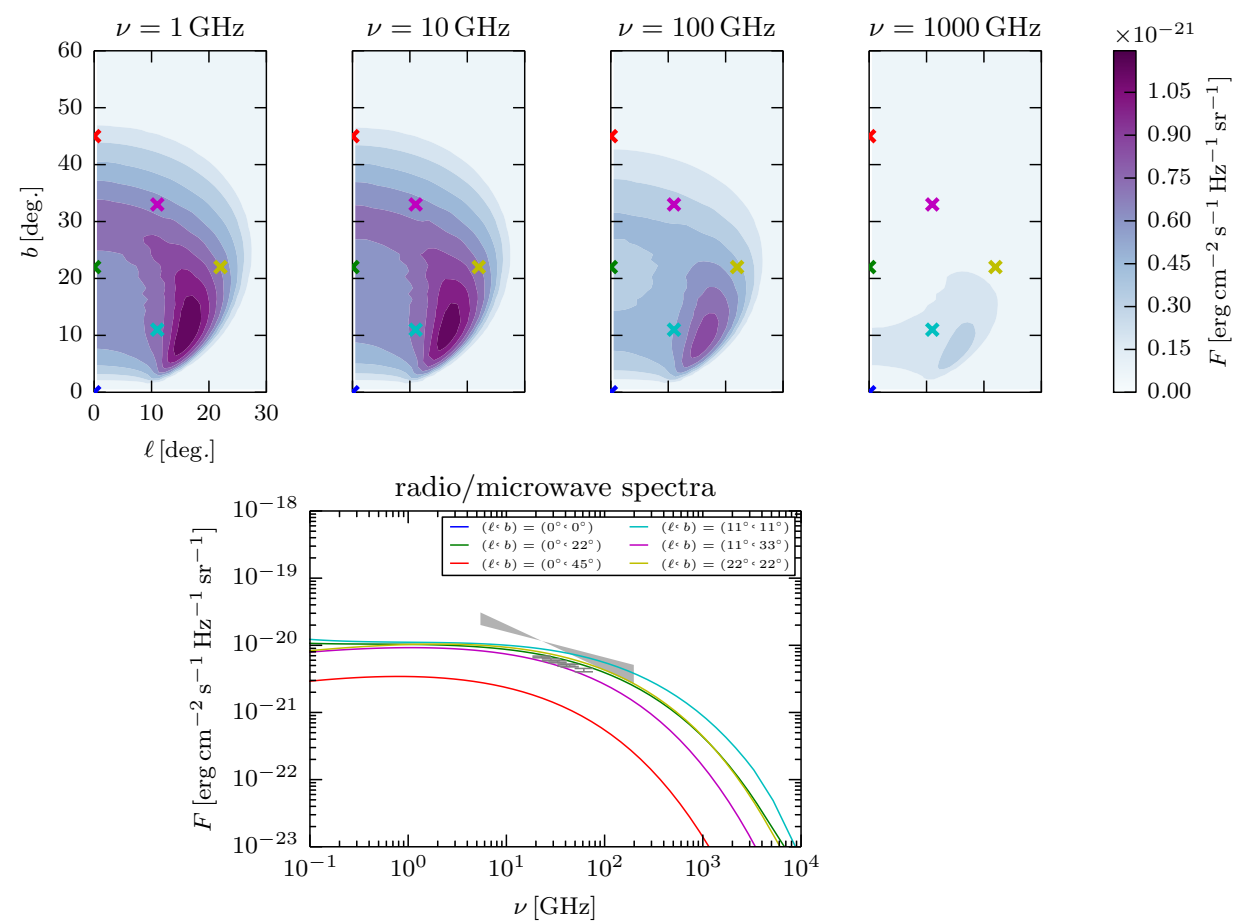

Figure 12: Top: Sky maps of the synchrotron intensity at 1, 10, 100 and $1000 \mathrm{GHz}$, produced by the same electrons shown in Fig. 10. Bottom: Synchrotron spectra for the different directions indicated in the legend and marked by the crosses in the lower panel of Fig. 10. The data are from Refs. [3] and [4].

\section{Summary}

We have reviewed the observations of the Fermi bubbles in gamma-rays, in particular their morphological and spectral properties. Most puzzling are their hard edges and their spectral uniformity which is a challenge to most models presented. Complementary information has been gained from X-ray data, but also from observations of UV absorption lines, both of which give (somewhat conflicting) bounds on the outflow velocities. Instead of reviewing all of the previous models in detail, we have focussed on a few exemplary ones, based on (M)HD simulations of jets and a Galactic wind. In our model, we focus on the acceleration of high-energy electrons due to turbulence in the presence of spatial diffusion and advection as well as Inverse Compton, synchrotron and adiabatic losses. We have presented three models differing in the assumptions about regions of enhanced turbulence and about (an)isotropy of spatial diffusion. We have presented one model with anisotropic diffusion deep inside and outside the bubbles, but almost isotropic diffusion in a shell around the bubbles' shock. This model is reproducing the observations, in particular the spectral and morphological properties in gamma-rays reasonably well while the microwave haze can also be explained from synchrotron emission of the same population of electrons.

\section{Acknowledgments}

The authors are grateful to Anna Franckowiak and Dmitry Malyshev for continued discussion. This work was supported by Danmarks Grundforskningsfond under grant no. 1041811001. PM was 
supported by DoE contract DE-AC02-76SF00515 and a KIPAC Kavli Fellowship. This research was funded in part by NASA through Fermi Guest Investigator grants NNH13ZDA001N.

\section{References}

[1] G. Dobler, D. P. Finkbeiner, I. Cholis, T. R. Slatyer, and N. Weiner, The Fermi Haze: A Gamma-Ray Counterpart to the Microwave Haze, Astrophys. J. 717 (2010) 825-842, [arXiv:0 910 . 4583].

[2] D. P. Finkbeiner, Microwave ism emission observed by wmap, Astrophys. J. 614 (2004) 186-193, [astro-ph/0311547].

[3] G. Dobler and D. P. Finkbeiner, Extended Anomalous Foreground Emission in the WMAP 3-Year Data, Astrophys. J. 680 (2008) 1222-1234, [arXiv:0712.1038].

[4] Planck Collaboration, P. A. R. Ade et al., Planck Intermediate Results. IX. Detection of the Galactic haze with Planck, Astron. Astrophys. 554 (2013) A139, [arXiv:1208.5483].

[5] M. Su, T. R. Slatyer, and D. P. Finkbeiner, Giant Gamma-ray Bubbles from Fermi-LAT: AGN Activity or Bipolar Galactic Wind?, Astrophys. J. 724 (2010) 1044-1082, [arXiv: 1005.5480 ].

[6] Fermi-LAT Collaboration, M. Ackermann et al., The Spectrum and Morphology of the Fermi Bubbles, Astrophys. J. 793 (2014), no. 1 64, [arXiv:1407. 7905 ].

[7] J.-M. Casandjian, I. Grenier, and f. t. F. L. A. T. Collaboration, High Energy Gamma-Ray Emission from the Loop I region, arXiv:0912.3478.

[8] M. Su and D. P. Finkbeiner, Evidence for Gamma-ray Jets in the Milky Way, Astrophys. J. 753 (2012) 61, [arXiv:1205.5852].

[9] D. Hooper and T. R. Slatyer, Two Emission Mechanisms in the Fermi Bubbles: A Possible Signal of Annihilating Dark Matter, Phys. Dark Univ. 2 (2013) 118-138, [arXiv:1302.6589].

[10] Fermi-LAT Collaboration, M. Ackermann et al., The Fermi Galactic Center GeV Excess and Implications for Dark Matter, Astrophys. J. 840 (2017), no. 1 43, [arXiv: 1704.0391 ].

[11] U. Keshet and I. Gurwich, Fermi bubble edges: spectrum and diffusion function, Astrophys. J. 840 (2017), no. 1 7, [arXiv:1611.0419].

[12] S. Veilleux, G. Cecil, and J. Bland-Hawthorn, Galactic winds, Ann. Rev. Astron. Astrophys. 43 (2005) 769-826, [astro-ph/0504435].

[13] P. Mertsch and S. Sarkar, Systematic effects in the extraction of the 'WMAP haze', arXiv:1004.3056.

[14] J. Kataoka et al., Suzaku observations of the diffuse $x$-ray emission across the fermi bubbles' edges, Astrophys. J. 779 (2013) 57, [arXiv:1310.3553].

[15] J. Kataoka, M. Tahara, T. Totani, Y. Sofue, Y. Inoue, S. Nakashima, and C. C. Cheung, Global Structure of Isothermal Diffuse X-ray Emission Along the Fermi Bubbles, Astrophys. J. 807 (2015), no. 1 77, [arXiv:1505.0593].

[16] M. J. Miller and J. N. Bregman, The Interaction of the Fermi Bubbles With the Milky Way?s hot gas Halo, Astrophys. J. 829 (2016), no. 1 9, [arXiv:1607.0490].

[17] F. Guo and W. G. Mathews, The Fermi Bubbles. I. Possible Evidence for Recent AGN Jet Activity in the Galaxy, ApJ 756 (Sept., 2012) 181, [arXiv:1103.0055]. 
[18] H.-Y. K. Yang, M. Ruszkowski, P. M. Ricker, E. Zweibel, and D. Lee, The Fermi Bubbles: Supersonic Active Galactic Nucleus Jets with Anisotropic Cosmic-Ray Diffusion, ApJ 761 (Dec., 2012) 185, [arXiv:1207.4185].

[19] K. C. Sarkar, B. B. Nath, and P. Sharma, Multiwavelength features of Fermi bubbles as signatures of a Galactic wind, MNRAS 453 (Nov., 2015) 3827-3838, [arXiv: 1505 . 0363].

[20] P. Mertsch and S. Sarkar, Fermi gamma-ray 'bubbles' from stochastic acceleration of electrons, Phys. Rev. Lett. 107 (2011) 091101, [arXiv:1104.3585].

[21] R. Blandford and D. Eichler, Particle acceleration at astrophysical shocks: A theory of cosmic ray origin, Phys. Rep. 154 (Oct., 1987) 1-75.

[22] P. Mertsch and V. Petrosian. in preparation. 